\title{
Squint-Free Beamsteering Demonstration using a Photonic Integrated Beamformer based on Optical Ring Resonators
}

\author{
M. Burla, M. R. H. Khan, \\ D. A. I. Marpaung, C. G. H. Roeloffzen \\ Telecommunication Engineering Group \\ University of Twente \\ Enschede, The Netherlands \\ m.burla@ewi.utwente.nl
}

\author{
P. Maat, K. Dijkstra \\ ASTRON \\ Dwingeloo, The Netherlands
}

\author{
A. Leinse, M. Hoekman, \\ R. Heideman \\ LioniX B.V. \\ Enschede, The Netherlands
}

\begin{abstract}
The squint-free beamsteering capability of a photonic integrated beamformer based on continuously tunable optical ring resonators has been demonstrated by showing, for the first time, its generated radiation patterns. The paper demonstrates a beamsteering of 16 degrees over an instantaneous bandwidth of at least $450 \mathrm{MHz}$.
\end{abstract}

\section{INTRODUCTION}

For many of the modern radio astronomy instruments wide instantaneous bandwidth is a characteristic of great interest. Some important science cases require high frequencies, broad bands and high beam shape stability, e.g. to improve sensitivity in continuum observations and survey speed for line observations (wide frequency coverage equates to wide velocity coverage, which means a larger 3-D volume of space surveyed per observation) [1]. An instantaneous bandwidth beyond several hundreds of $\mathrm{MHz}$ can be a difficult requirement for a phased array antenna (PAA) employing a traditional electronic beamformer. The limited speed of the $\mathrm{A} / \mathrm{D}$ converters and of the processing devices is the limiting factor towards broader bands for digital beamformers, which are widely used in radio astronomy for their flexibility in terms of beam shape control and interference nulling [2]. In turn, for the case of analog beamforming, the performance of the constituting components, mainly combiners and delay elements, limits the bandwidth of the total system. Photonic technology allows to overcome this limit by providing wideband and continuously tunable delay elements and combiners, as demonstrated by several authors in the last years $[3,4]$.

We propose an optical beamformer chip realized in a lowloss, silica-based optical waveguide technology, employing Mach-Zehnder Interferometers (MZIs) as tunable combiners, and optical ring resonators (ORRs) as seamlessly tunable true time delay units, basic building blocks for the so-called integrated optical beamforming network (OBFN), providing a

This work is within the framework of the MEMPHIS project, for which the authors gratefully acknowledge the support of the Smart Mix Programme of the Netherlands Ministry of Economic Affairs and the Netherlands Ministry of Education, Culture and Science. high precision in terms of beam shape control and reproducibility. The signals received from the individual antenna elements of the PAA are first amplified and then, using an array of electro-optical modulators, brought into the optical domain where they are individually delayed and combined, before being converted back to electrical domain via a photodetector. In [5] we first showed the broadband optical group delay generation by the OBFN, then a complete characterization of the RF-to-RF properties of an optical beamforming system for airborne PAA applications [6]. In this paper, for the first time, we demonstrate the squint-free beamsteering capability of this system by displaying the measured radiation patterns obtained by integrating the OBFN with an antenna array for radioastronomy.

\section{RING RESONATOR-BASED OBFN}

In our system, the broadband delay is realized with an optical ring resonator (ORR), which consists on a straight waveguide coupled to a race-track-shaped line of length $L$ and corresponding round trip time $T$. The coupling element is a tunable MZI: changes in the phase of one of its branches have the effect of modifying the power coupling coefficient $\kappa$, and thus the quality factor of the resonator, which directly relates to the slope of the phase characteristic around the resonance frequency. The resulting phase response of the ORR can then be tuned in such a way to approximate the desired linear phase response of an ideal true time delay line, with an error $\varepsilon$ below a specified amount, over a wide band. An example is depicted in Fig. 1. The phase characteristic of a line with a delay of $2.36 T$ is approximated by the ORR phase response. For this delay, the phase error is below $\pi / 16$ on a band of $23.85 \%$ of the FSR (the inverse of the round-trip time T). For a typical $F S R$ of $20 \mathrm{GHz}$ (corresponding to a $T$ of $50 \mathrm{ps}$ ) the ORR would then give a delay of 118.13 ps over a band of $4.77 \mathrm{GHz}$. Increasing the delay, the bandwidth reduces and vice versa (Fig. 2) 


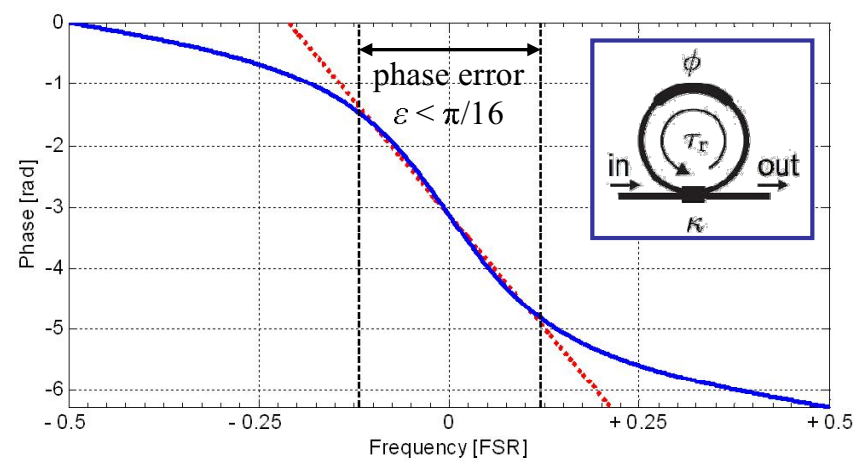

Figure 1. Phase response comparison: ORR (bold) vs. ideal delay line (dotted). In the inset: ORR schematic.

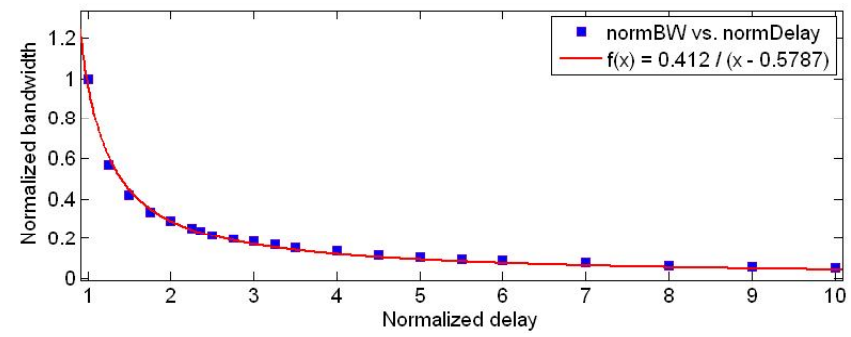

Figure 2. Normalized bandwidth versus normalized delay and fitting curve

This bandwidth can be further increased by simply cascading multiple ring resonator units and tuning them in such a way that the resonance, thus the phase transition, occurs at a different frequency. This frequency tuning is made possible by adding a tunable phase shifting element on the ring waveguide section (see inset, Fig. 1). For a complete description of the ORR delay elements see [7].

The devices described in the previous subsection can be used as building blocks to assemble a complete optical beam forming network, whose schematic layout is shown in Fig. 3. Each of the 16 optical inputs (left) carries the signal originating from a single antenna element in the $4 \times 4$ subarray of the demonstrator antenna tile which will be described in Section III. This architecture allows to control the individual magnitude and phase weights of each antenna element by employing the tunability of combiners and delay units [7].

The OBFN illustrated in Fig. 3 has been realized by LioniX B.V. on a low-loss, Si-based substrate (TriPleX ${ }^{\mathrm{TM}}$ ), as described in detail in [8]. The tunable elements are controlled with a thermo-optical mechanism, where resistive heaters are driven with DC voltages set with high accuracy and repeatability by a microprocessor and a 14 bit $\mathrm{D} / \mathrm{A}$ converter. The chip is mounted on a thermo-conductive copper base, connected to a heat dissipator and kept to a stable temperature by a simple feedback system, based on resistive temperature sensors and Peltier elements [8].

\section{SYSTEM SETUP}

The optical beamformer described in Section II has been integrated in a complete system by ASTRON and University of Twente within the framework of SKADS (Square
Kilometer Array Design Studies). A $4 \times 4$ subarray of the EMBRACE (Electronic Multi-beam Radio Astronomy Concept) antenna tile has been modified by adding a photonic section after the low-noise amplifier (LNA) stages, in place of the electronic back-end, by keeping the same structure for the antenna elements and the LNAs. This system is expected to give an operating instantaneous bandwidth a factor 10 higher than the one employing the electronic beamformer, for all observation angles [9].

In this first measurement stage, only a linear subarray of 4 antenna elements has been connected to the optical beamformer. In more detail, the output of each low noise amplifier has been fed to a Photline ${ }^{\circledR}$ MXAN-LN-10 MachZehnder Modulator (MZM), mounted on the same PCB as the LNAs. Those modulators are specifically designed for analog links and antenna applications, and have a $v_{\pi} \mathrm{RF}$ of $5 \mathrm{~V}$ and an insertion loss of approximately $4 \mathrm{~dB}$. The optical input to the MZMs is provided by a high optical power $(100 \mathrm{~mW}) \mathrm{EM} 4^{\circledR}$ distributed feedback laser and a 1-to-16 optical splitter array by Gould Fiber Optics ${ }^{\circledR}$. The modulator output signals are then injected into the OBFN inputs to be delayed and combined as desired. The OBFN output is then fed to a high speed detector $\left(13 \mathrm{GHz}\right.$ Agere $^{\circledR} 2560 \mathrm{~A}$, responsivity $\left.0.8 \mathrm{~A} / \mathrm{W}\right)$. The schematic in Fig. 4 gives an overview of the setup used for the radiation pattern measurements. Two different lasers were used during the tests: a high optical power $(100 \mathrm{~mW}) \mathrm{EM} 4^{\circledR}$ distributed feedback laser has been used as sweeping source for the purpose of tuning the OBFN; a high-stability ANDO ${ }^{\circledR}$ AQ4321D laser, tuned in the center of the optical delay band, has been used in conjunction with a Keopsys ${ }^{\circledR}$ KPS-BT-C21PB-FA erbium-doped fiber amplifier (EDFA) during the radiation pattern measurements. This was done to minimize the laser wavelength instabilities previously experienced when using the same EM4 ${ }^{\circledR}$ high-power laser for both purposes: changing the operating mode of the laser required stabilization times in the order of hours.

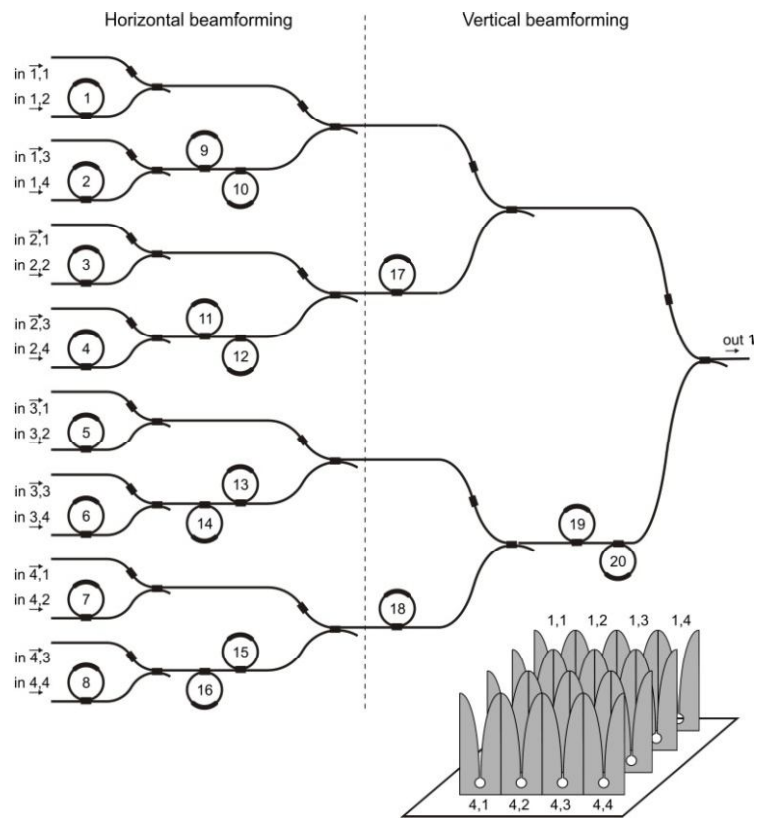

Figure 3. $16 \times 1 \mathrm{OBFN}$ and corresponding $4 \times 4$ array schematic 


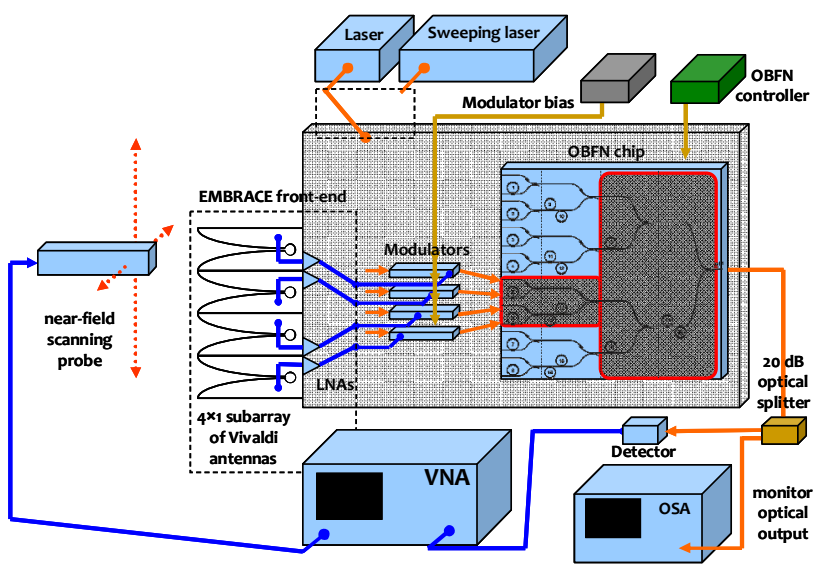

Figure 4. Setup used in the radiation pattern measurements

Note that in the setup shown in Fig. 4 and described above, in conjunction with the integrated beamformer chip, several fiber-based components are employed: splitters, MZMs and SOAs (not used in this test). As a consequence, several meters of fiber can be found between the first optical splitting point and the inputs to the beamformer chip, where the optical combining occurs. This creates an important practical issue related to coherent optical combining, which is required at all times in order to keep the maximum optical power at the detector. This is needed to maximize the signal to noise ratio of the detected RF output signal [10]. Optical phase synchronization has been achieved by using a careful design of the mechanical structure for the demonstrator, where the fibers have been given a proper mechanical support to avoid undesired vibrations and their temperature kept stable by using a proper casing (Fig. 5, 6).

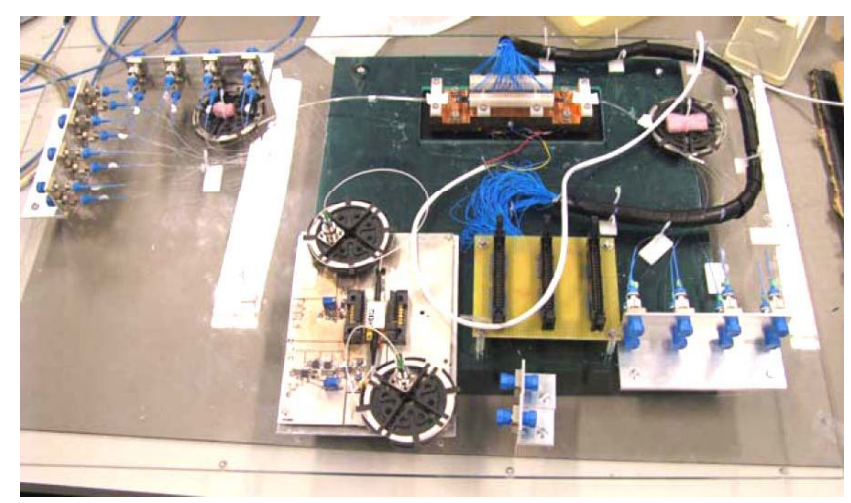

Figure 5. OBFN chip (center, top) with input (top left) and output (bottom right) fiber connectors. Visible also the bundle of wires for OBFN control.

\section{ANTENNA MEASUREMENTS}

The antenna pattern measurements have been carried out in the Antenna Test Range in ASTRON. The test range is equipped with a near-field scanner by NSI Nearfield Systems ${ }^{\circledR}$. The whole setup has been mounted vertically, in such a way that the broadside beam direction coincides with the axis of the near field scanning probe. For the way in which the EMBRACE tile is realized, the E-plane forms an angle of 45 degrees with the scanning directions: this has been accounted for via software in the process of far-field extraction from the near-field measurements. As a starting test, only a subarray of 2 antennas has been measured. The neighboring elements have also been connected to the corresponding correctly biased LNAs in order to properly account for the effects of mutual coupling and avoid pattern distortions.

The operating frequency band of the EMBRACE tile is 400 to $1550 \mathrm{MHz}$. The antenna element spacing is $12.5 \mathrm{~cm}$. Due to the low operating frequency of the array and the number of antennas limited to 2 , we expected a very broad beam pattern: the simulation gives a position of the first nulls at \pm 55.6 degrees from the broadside direction (Fig. 7). Such a broad main lobe makes it difficult to measure small beamsteering angles. For this reason, a clever expedient has been used to improve the readability of the measured diagrams: instead of using two neighboring elements, the pattern originated by two antennas spaced $3 / 2 \lambda$ has been measured. This created two evident grating lobes, separated by the main one by deep nulls of radiation at just 15.9 degrees from the broadside direction (Fig. 7). This allowed a much clearer view of the squint-free beamsteering capability of the system, for the starting test with only two antenna elements and limited steering angles.

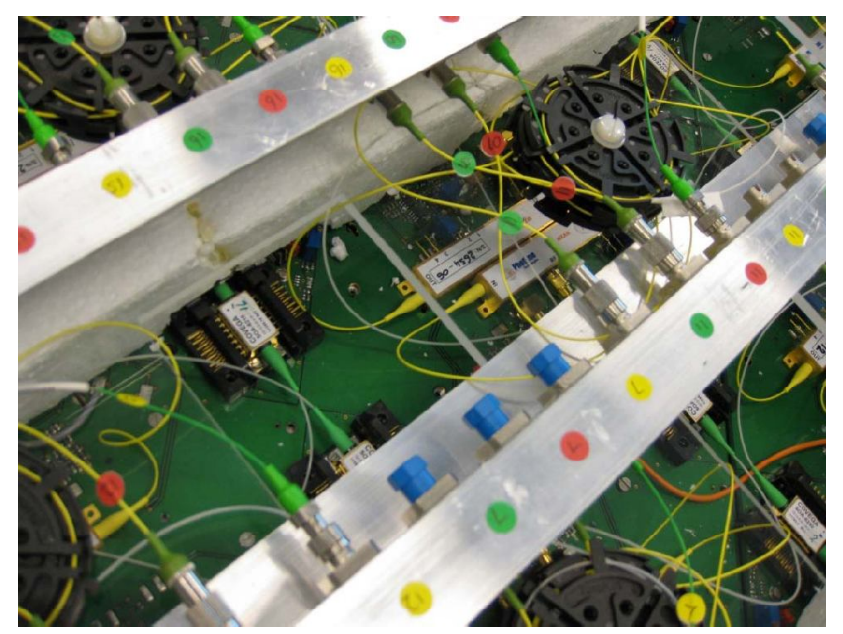

Figure 6. Detail of the back side of the antenna tile. The discrete optical components are mounted on the same PCB as the LNAs. Note the reels and the panels for the mechanical support of fibers and connectors.

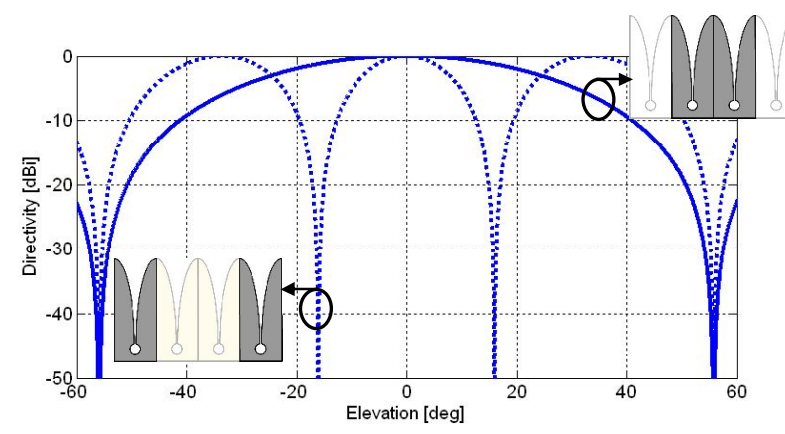

Figure 7. Simulated radiation pattern for 2 elements spaced half-wavelength (bold line) and 1.5 wavelengths (dotted line): deliberate generation of grating lobes for accurate measurements of low beamsteering angles. 

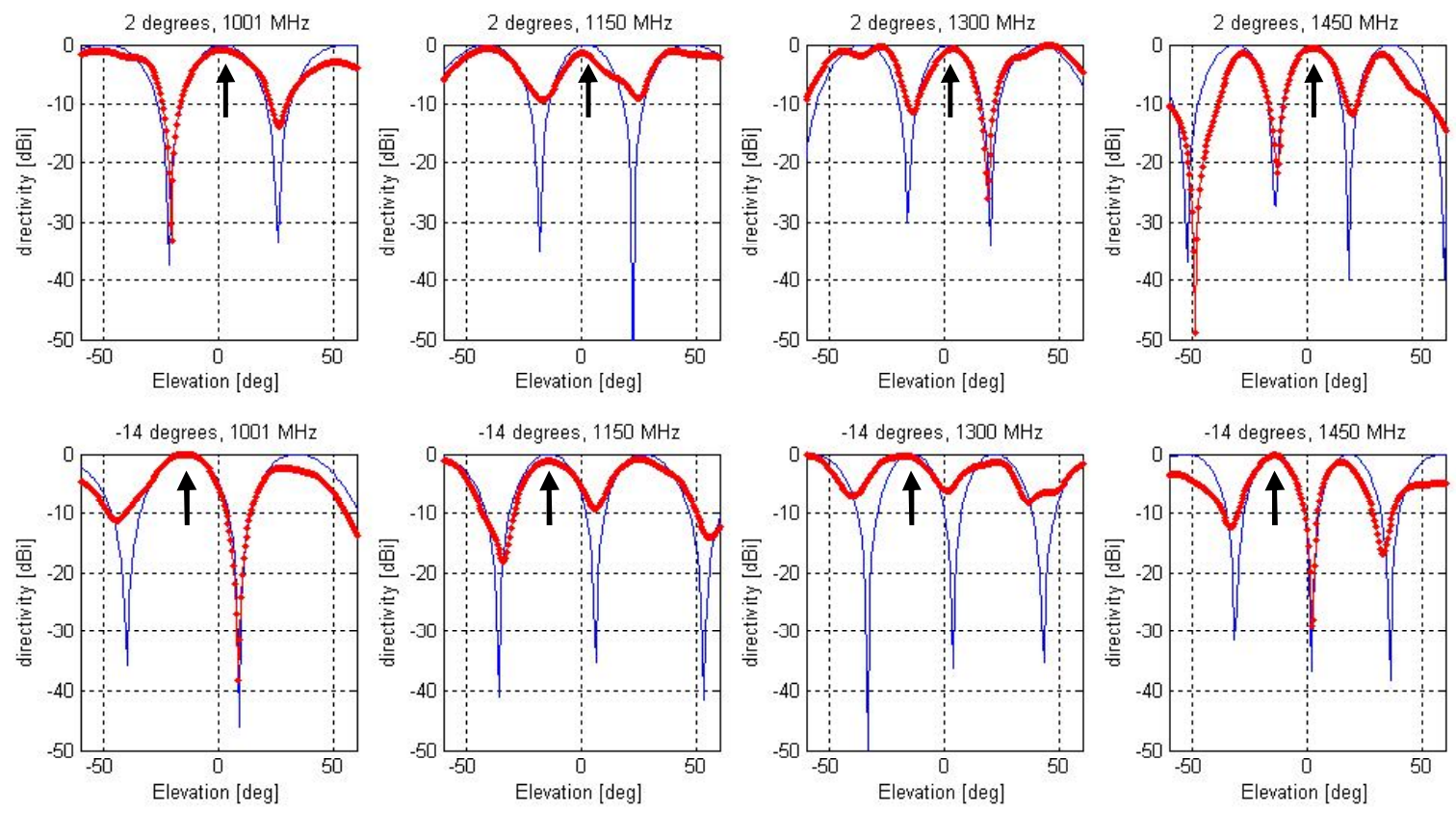

Figure 8. Measured (red) vs simulated (blue) radiation patterns (45 degrees rotated E-plane) for +2 degrees (top) and -14 degrees (bottom) steering angles for the main beam, at four frequencies (left to right: $1001 \mathrm{MHz}, 1150 \mathrm{MHz}, 1300 \mathrm{MHz}, 1450 \mathrm{MHz}$ ). Note the absence of beam squinting for the main lobe (indicated by the arrows) over the whole frequency range. The slight beam shift at $1300 \mathrm{MHz}$ can be attributed to a temporary laser instability experienced during the tests, which might have shifted the modulating signal at the edge of the ORR delay band.

Fig. 8 shows the measured radiation patterns compared to their MatLab ${ }^{\circledR}$ simulation, at four different frequencies: $1001 \mathrm{MHz}, 1150 \mathrm{MHz}, 1300 \mathrm{MHz}$ and $1450 \mathrm{MHz}$. For comparison, a system that would employ phase shifters instead of TTD units (to create a beam at -14 degrees at $1001 \mathrm{MHz}$ ), would give a beam direction error of 1.84 , 3.26 and 4.38 degrees at the last three frequencies, respectively. Unfortunately the measurement could not be extended to lower frequencies due to the cutoff frequency of the near-field waveguide probe. Being this the limiting factor, still we would expect the squint-free beam pointing to be maintained for frequencies as low as $400 \mathrm{MHz}$, for a total instantaneous bandwidth beyond $1 \mathrm{GHz}$.

\section{CONCLUSIONS}

The capability of an ORR-based optical beam forming network to generate beamsteering without frequency squint has been demonstrated via radiation pattern measurements. As a proof-of-principle, we obtained a squint-free steering of more than 15 degrees over a band of $450 \mathrm{MHz}$. The ongoing work aims to extend the measurements shown in this paper to a setup with 4 antenna elements, and with higher steering angles. In parallel, we are working towards a fully integrated demonstrator, with no fiber-based components: this will completely solve the optical phase instability issues mentioned in Section III.

\section{REFERENCES}

[1] G. Heald, T. Oosterloo, and M. Verheijen, "The science case for APERTIF," BYU Phased Array Antenna Workshop, Provo, Utah, 3-5 May 2010.
[2] B. D. Jeffs and K. F. Warnick, "Bias corrected PSD estimation for an adaptive array with moving interference," IEEE Trans. on Signal Processing, vol. 56, no. 7, pp. 3108-3121, July 2008.

[3] N. A. Riza, Selected Papers on Photonic Control Systems for Phased Array Antennas, ser. SPIE Milestone. New York: SPIE, Jun. 1997, vol. MS 136.

[4] A. J. Seeds and K. J. Williams, "Microwave photonics," J. Lightwave Technol., vol. 24, no. 12, pp. 4628-4641, Dec. 2006.

[5] L. Zhuang, C. G. H. Roeloffzen, R. G. Heideman, A. Borreman, A. Meijerink, W. C. van Etten, "Single-chip ring resonator-based $1 \times 8$ optical beam forming network in CMOS-compatible waveguide technology," IEEE Photon. Technol. Lett., vol. 19, no. 15, Aug. 2007.

[6] L. Zhuang, M. Burla, C. G. H. Roeloffzen, A. Meijerink, D. A. I. Marpaung, M. R. H. Khan, W. C. van Etten, A. Leinse, M. Hoekman, R. G. Heideman, "RF-to-RF characterization of a phased array receive antenna steering system using a novel ring resonator-based integrated photonic beamformer," in Proc. IEEE MWP 2009, Valencia, Spain, Oct. 14-16, 2009.

[7] A. Meijerink et al., "Novel ring resonator-based integrated photonic beamformer for broadband phased array receive antennas-Part I: Design and performance analysis," J. Lightwave Technol., vol. 28, no. 1, pp. 3-18, Jan. 2010 .

[8] L. Zhuang et al., "Novel ring resonator-based integrated photonic beamformer for broadband phased array receive antennas-Part II: Experimental prototype," J. Lightwave Technol., vol. 28, no. 1, pp. 19-31, Jan. 2010.

[9] G. W. Kant, P. D. Patel, S. J. Wijnholds, M. Ruiter, E. van der Wal, "EMBRACE: A multi-beam 20,000-element radio astronomical phased array antenna demonstrator," IEEE Trans. on Antennas and Propag., Special Issue: Antennas for Next Generation Radio Telescopes, in press.

[10] N. M. Froberg, E. I. Ackerman, and C. H. Cox III, "Analysis of signal to noise ratio in photonic beamformers," in Proc. IEEE Aerospace Conf. 2006, Big Sky, MT, Mar. 4-11, 2006. 